\title{
Evaluación y repercusiones perinatales del riesgo materno-fetal
}

\author{
Dres. Antonio Soto-Yances*, Elsy A. de Merlano**
}

\begin{abstract}
RESUMEN. Se aplicó un formulario, diseñado y elaborado por un grupo de trabajos anteriores, a un total de 100 pacientes que fueron hospitalizadas en el Hospital de Maternidad Rafael Calvo C., entre los meses de julio a diciembre de 1989.

El mencionado formulario fue creado para permitir la clasificación de estas pacientes con patologías de bajo, mediano y alto riesgo, utilizando la siguiente puntuación: de 1 a 4 puntos, pacientes de bajo riesgo; de 5 a 9 puntos, pacientes de mediano riesgo, y de 10 o más puntos, pacientes de alto riesgo.

De acuerdo con lo anterior se observó que de las 100 pacientes estudiadas el $90 \%$ fueron clasificadas como de alto riesgo, un $8 \%$ como de mediano riesgo y un $2 \%$ como de bajo riesgo.

Entre los antecedentes y/o patologías actuales de bajo riesgo lo más destacable fue: madre soltera (35 casos), falta de asistencia prenatal ( 18 casos), primípara menor o igual a 18 años o mayor o igual a 35 años (14 casos).

Entre la patología de mediano riesgo lo más significativo fue: anemia menor de 10 grs (31 casos) e infección urinaria (14 casos). En lo que hace referencia a pacientes con patologías de alto riesgo lo más importante fue: preeclampsia severa (34 casos), hemorragias de la segunda mitad del embarazo (24 casos), prematuridad ( 24 casos), ruptura prematura de membranas ( 21 casos), eclampsia (6 casos).

En general las patologías más encontradas fueron: preeclampsia severa, anemia menor de 10 grs, hemorragia de la segunda mitad del embarazo, prematuridad y ruptura prematura de membranas.

En cuanto al tipo de parto, de las 100 pacientes, a 52 de ellas se les dio parto abdominal y a las 48 restantes se les dio parto vaginal.

Las cifras de mortalidad fetal fueron las siguientes: 18 muertes, todas producto de embarazos de alto riesgo.
\end{abstract}

PALABRAS CLAVES: Alto riesgo, preeclampsia, Ruptura prematura de membrana.

SUMMARY: a formulary was applied, designed and elaborated by a group of former works, for a total of 100 patients hospitalized in the maternity hospital "Rafael Calvo" during the months july to december 1989.

The mentioned formulary was created to allow the classification of these patients with low, moderate and high risk pathologies using the following punctuation: 1 to 4 points, low riks patients; 5 to 9 points, moderate risk patients and 10 or more points high risk patients.

According to the above, it was observed that of the 100 studied patients, the $90 \%$ were classified as of high risk, an $8 \%$ as of moderate risk and a $2 \%$ as of low risk.

Into the previous and/or actual pathologies of low riks, it is outstanding: unmarriea mother (35 cases) lack of prenatal assistance ( 18 cases) primapara, younger or equal to 18 years old or older or equal to 35 years old (14 cases).

In the moderate risk pathology, the outstandig was: anemia less of 10 grames ( 31 cases) and urinary infecion ( 14 cases). In relation with high risk pathologies, the outstang was: severe pre-eclampsia ( 34 cases) hemorrhage in the second half of pregnancy ( 24 cases) premature ( 24 cases), premature breaking of membrane ( 21 cases) eclampsia (6 cases).

Generally, the outstanding pathologies were: severe preeclampsia, anemia less than 19 grams, hemorrhage of the second half of pregnancy, premature breaking of membranes.

In relation with kind of child birth of the 100 patients, 52 were given with abdominal childbirth and the other 48 had vaginal childbirth.

The fetal death figures were the following: 18 deaths all product of high risk pregnancy.

KEY WORDS: High risk, pre-eclampsia, premature breaking of membrane.

\section{Introducción}

En los servicios de urgencias gineco-obstétricos del mundo siempre ha existido la dificultad de poder clasificar de manera rápida y oportuna a aquellas pacientes embarazadas que estén presentando algún tipo de patología que pueda

\footnotetext{
* Profesor Titular, Jefe del Dpto. de Ginecología y Obstetricia, Universidad de Cartagena, Facultad de Medicina.

** Residente III, Dpto. de Gineco-Obstetricia.
}

ser, en determinado momento, considerada como de bajo, mediano y alto riesgo.

Es por eso que en ciertos países más avanzados científica y socialmente (2) que el nuestro, y aún algunos que están en un nivel parecido al que tenemos ahora (4) se ha intentado diseñar algún mecanismo por medio del cual se pueda detectar precozmente a ese grupo de pacientes embarazadas con riesgo. Del esfuerzo anterior han surgido algunos cuestionarios o formularios, algunos más prácticos que otros $(2,4$, 
8), que en menor o mayor grado cumplen el propósito para el cual fueron diseñados.

La dificultad estriba en el hecho de que lo que es aplicable y práctico en otras latitudes, no lo es siempre en este contexto. Esta es la razón primordial por la cual en Colombia se ha intentado, en forma aislada o regional, la elaboración de un formulario propio que se ajuste a la realidad y necesidades (5).

En el caso concreto del Hospital de Maternidad Rafael Calvo C., de Cartagena, un grupo de trabajo integrado por estudiantes de 5o. año, residentes y docentes de la Facultad de Medicina de la Universidad de Cartagena, se entregaron a la ardua labor de intentar el diseño de un formulario de rápida y fácil aplicación que pudiese ser utilizado en pacientes embarazadas, con especial énfasis en aquellas que se encuentren en el tercer trimestre de la gestación (5). Esto permitiría la oportuna detección de aquellas pacientes de riesgo, para poder planear y administrar en forma precoz un manejo adecuado, todo lo cual se reflejaría como lógica consecuencia en la disminución de la morbi-mortalidad materna y fetal.

\section{Justificación}

En los servicios de urgencias y consulta externa prenatal del Hospital de Maternidad Rafael Calvo C. se ha observado a través de entrenamiento, que si bien existen criterios definidos para hospitalizar a las pacientes, éstos no se encuentran unificados para determinar el grado o tipo de riesgo durante el embarazo.

Con esta inquietud se decide elaborar un formulario que fue el motivo del trabajo anterior y que se aplicará para determinar, de manera objetiva, su valor en la ayuda a la paciente embarazada.

\section{Objetivos generales}

1. Aplicar el formulario experimental para evaluar en forma cuantitativa el grado de riesgo materno-fetal en el embarazo.

2. Que este documento forme parte de toda historia clínica obstétrica elaborada en el Hospital de Maternidad Rafael Calvo C.

3. Evaluar la eficiencia, eficacia y efectividad del formulario.

\section{Objetivos específicos}

1. Demostrar que la aplicación temprana y adecuada de este formulario, contribuye a disminuir la morbi-mortalidad materna y perinatal.

2. Clasificar un embarazo en bajo, mediano o alto riesgo de acuerdo al puntaje obtenido, para brindar una atención médica dirigida e individual.

\section{Hipótesis}

A pesar de ser considerado el embarazo como un estado fisiológico, hay diversas circunstancias patológicas que se presentan durante él, que obligan a establecer un seguimiento más detallado y un manejo más específico que, de no llevarse a cabo, lesionaría altamente la salud de la madre y/o del fruto concebido.

Nuestro propósito es clasificar a esa paciente embarazada que necesita manejo especial, de acuerdo con parámetros establecidos y reconocidos por la ciencia médica, con el fin de disminuir la morbi-mortalidad materno-fetal.

Demostrar que la aplicación de este formulario permite establecer los niveles de riesgo, con el fin de tomar conductas adecuadas y específicas de acuerdo con cada caso en particular.

\section{Material y métodos}

Se tomó una muestra de 100 pacientes hospitalizadas en el Hospital de Maternidad Rafael Calvo C., de Cartagena, en el servicio de cuidados especiales, durante el período de tiempo comprendido entre los meses de julio a diciembre de 1989, quienes presentaban embarazo de riesgo.

Es un trabajo retrospectivo-prospectivo, longitudinal, clínico, cuasiexperimental. El análisis de sus datos fue realizado a partir de información obtenida de las historias clínicas de ingreso y/o por entrevistas directas.

Este estudio constituirá un ensayo previo de cuya aplicación saldrá la aceptación o modificación de la hipótesis, caso este último que conllevaría a la elaboración de un nuevo diseño del formulario, si no se demuestra plenamente dicha hipótesis.

Se utilizaron 100 formularios, atendiendo el número de pacientes correspondientes a la muestra.

Dicho formulario consta de cinco secciones, distribuidas así:

1. Identificación personal.

2. Antecedentes personales y gineco-obstétricos.

3. Patologías de mediano riesgo durante el embarazo.

4. Patologías de alto riesgo durante el embarazo.

5. Datos sobre el parto, producto y egreso de la madre.

\section{Instructivo del formulario} ción.

La primera sección es para recolectar datos de identifica-

En la segunda parte se consignan los antecedentes médicos y gineco-obstétricos y algunos datos personales actuales.

En el tercer acápite se anotan las patologías del embarazo que se consideran de mediano riesgo.

En la cuarta sección se registran las patologías del embarazo consideradas de alto riesgo.

La quinta sección registra el resultado del parto. Se especifica el tipo de parto y el producto fetal, el estado del recién nacido, la madre y las complicaciones subsiguientes.

La valoración materno-fetal se basa en el puntaje dado en la segunda, tercera y cuarta secciones.

A los ítems ubicados en la sección dos, se les da un puntaje de 1 a cada uno de los positivos.

En la sección tres a cada ítem positivo se le asignan 5 puntos.

En la cuarta sección se asignan 10 puntos por cada ítem positivo.

Realizada la suma de puntos de las tres secciones se clasifica el riesgo materno-fetal de la siguiente manera:

1. BAJO RIESGO ......................... 1 a 4 puntos.

2. MEDIANO RIESGO ................. 5 a 9 puntos.

3. ALTO RIESGO …................. 10 o más puntos.

Se sugiere que la conducta a seguir de acuerdo al grado de riesgo materno-fetal obtenido sea: 
1. BAJO RIESGO Observación.

2. MEDIANO RIESGO ........... Evaluación sistemática.

3. ÁLTO RIESGO .................. Conducta agresiva.

\section{Resultados}

Estudios de las pacientes. Se estudiaron 100 pacientes, encontrándose que el $90 \%$ de ellas eran de alto riesgo, un $8 \%$ de mediano riesgo y un $2 \%$ de bajo riesgo (Tabla 1 ).

Antecedentes médicos y gineco-obstétricos. Se analizaron por separado cada una de las pacientes encontrándose que los antecedentes médicos y gineco-obstétricos y datos personales actuales más frecuentes fueron: feto muerto o prematuridad anteriores, 2 casos; preeclampsia anterior, 2 casos; anemia menor de $10 \mathrm{grs,} \mathrm{un} \mathrm{caso;} \mathrm{primípara} \mathrm{menor}$ o igual a 18 años o mayor o igual a 35 años, 14 casos; paridad mayor o igual a seis, 7 casos; falta de asistencia prenatal, 18 casos; madre soltera, 35 casos; inercia y/o falta de progreso, 3 casos; otros, 16 casos (Tabla 2).

Patologías. Con respecto a las patologías de mediano riesgo, las más frecuentes fueron: anemia menor de $10 \mathrm{grs,}$ 31 casos; cirugía uterina o vaginal anterior, 3 casos; infección urinaria, 14 casos; preeclampsia leve, 4 casos; hipertensión arterial, 10 casos; otros, 17 casos (Tabla 3).

Las patologías de alto riesgo más frecuentemente encontradas fueron: preeclampsia severa, 34 casos; ruptura prematura de membranas, 21 casos; modificación de la frecuencia

Tabla 1

\begin{tabular}{|lc|}
\hline \multicolumn{1}{|c|}{ Clasificación de patologías } & No. de pacientes \\
\hline Alto riesgo $\geqslant$ de 10 & 90 \\
Mediano riesgo $(5-9)$ & 8 \\
Bajo riesgo & 2 \\
\hline
\end{tabular}

\section{Tabla 2
ANTECEDENTES PERSONALES}

\begin{tabular}{|lr|}
\hline \multicolumn{1}{|c|}{ Antecedentes personales } & No.de pacientes \\
\hline Feto muerto o prematuridad anterior & 2 \\
Pre-eclampsia anterior & 3 \\
Anemia $<10$ gr & 1 \\
Primigesta $\leqslant 18$ años $0 \geqslant 35$ años & 14 \\
Paridad $\geqslant 6$ & 7 \\
Falta de asistencia antenatal & 18 \\
Madre soltera & 35 \\
Inercia y/o falta de progreso & 3 \\
Narcóticos - Alcoholismo - Tabaquismo $>15$ & 0 \\
Otros & 16 \\
\hline
\end{tabular}

Tabla 3

PATOLOGIA DE MEDIANO RIESGO ASOCIADA A EMBARAZO ACTUAL

Patología

Anemia $<10$ gr

Cirugía uterina o vaginal anterior

Infección urinaria

Pre-eclampsia leve

Hipertensión arterial

Otras
Tabla 4

PATOLOGIA DE ALTO RIESGO

ASOCIADA A EMBARAZO ACTUAL

\begin{tabular}{|lc|}
\hline \multicolumn{1}{|c|}{ Patología } & No.de pacientes \\
\hline Pre-eclampsia severa & 34 \\
Ruptura prematura de membranas & 21 \\
Modificación de la F.C.F. & 13 \\
Prematuridad & 24 \\
Hemorragia de la segunda mitad del embarazo & 24 \\
Postmadurez & 0 \\
Eclampsia & 6 \\
Hidramnios & 2 \\
Otros & 19 \\
\hline
\end{tabular}

Tabla 5

RELACION ENTRE GRADO DE RIESGO

MATERNO FETAL Y TIPO DE PARTO

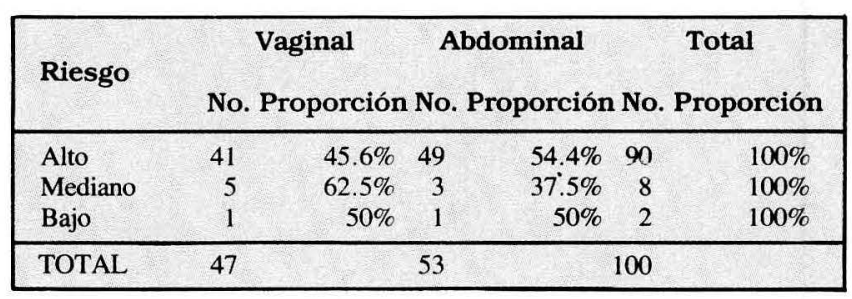

\section{REPRESENTACION DE LA TABLA 5}

\section{VAGINAL}

$\square$ ABDOMINAL
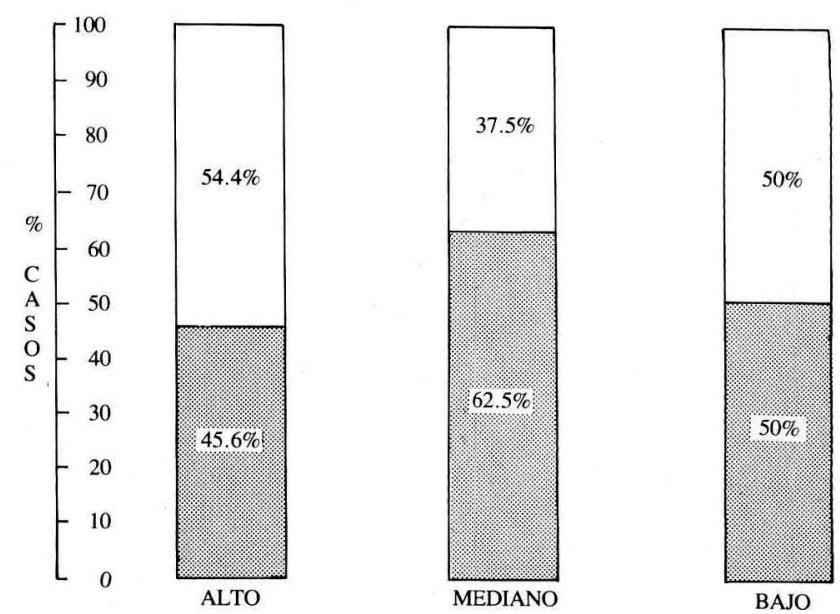

cardíaca fetal, 13 casos; prematuridad, 24 casos; eclampsia, 6 casos; hidramnios, 2 casos; otras, 19 casos (Tabla 4).

Tipo de parto. En cuanto al tipo de parto dado a las pacientes de alto riesgo se observó que se les practicó parto abdominal a un $54.4 \%$, en las de mediano riesgo a un $37.5 \%$ $y$ en las de bajo riesgo a un 50\% (Tabla 5).

Mortalidad fetal. Con relación a la mortalidad fetal se observó que entre las pacientes de alto riesgo hubo 18 casos de recién nacidos muertos (20\%); en las de mediano, al igual que en las de bajo riesgo, no hubo mortalidad (Tabla 6).

Apgar. En lo referente al apgar se observó que en las pacientes de alto riesgo se obtuvieron recién nacidos con apgar 
UNIVERSIDAD DE CARTAGENA

DEPARTAMENTO DE OBSTETRICIA Y GINECOLOGIA

UNIDAD DE ALTO RIESGO PERINATAL

FORMULARIO PARA LA VALORACION DEL RIESGO EN EL EMBARAZO

$$
\text { NOMBRE }
$$

DIRECCION

G. $\square$ P. $\square$ A. $\square$
PREMATURO $\square$
PRODUCTO MUERTO

PRODUCTO MUERTO

MUERTE NEONATAL.

HIJOS VIVOS
EDAD

-FECHA

U. M.

F. E. P.

EDAD GESTACIONAL

FECHA DE INGRESO

NATURAL

NATURAL

\section{PROCEDENTE RESIDENTE}

No DE H.C.

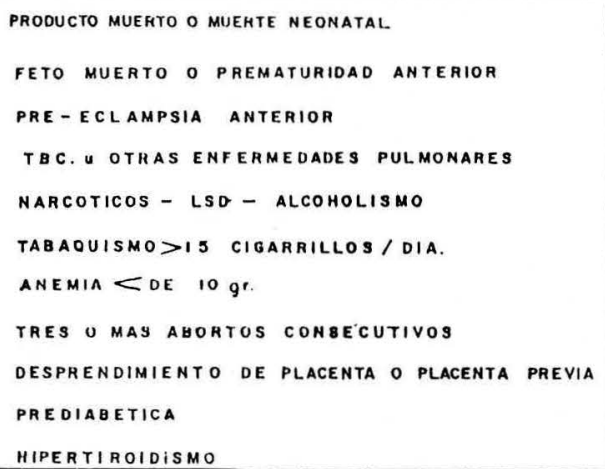

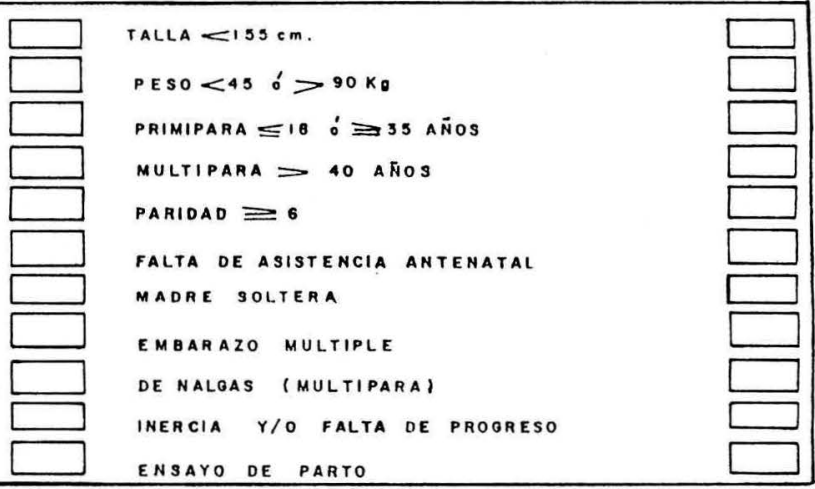

INPERTILIOAD PREVIA
EMBARAZO MULTIPLE
HIPERTENSION ARTERIAL PREVIA
INFECCION URINARIA
PRE-ECLAMPSIA LEVE DIASTOLICA CIOO
PODALICA EN NULIPARA IIAS INFECCIONES
PLACENTA PREVIA NO SANGRANTE

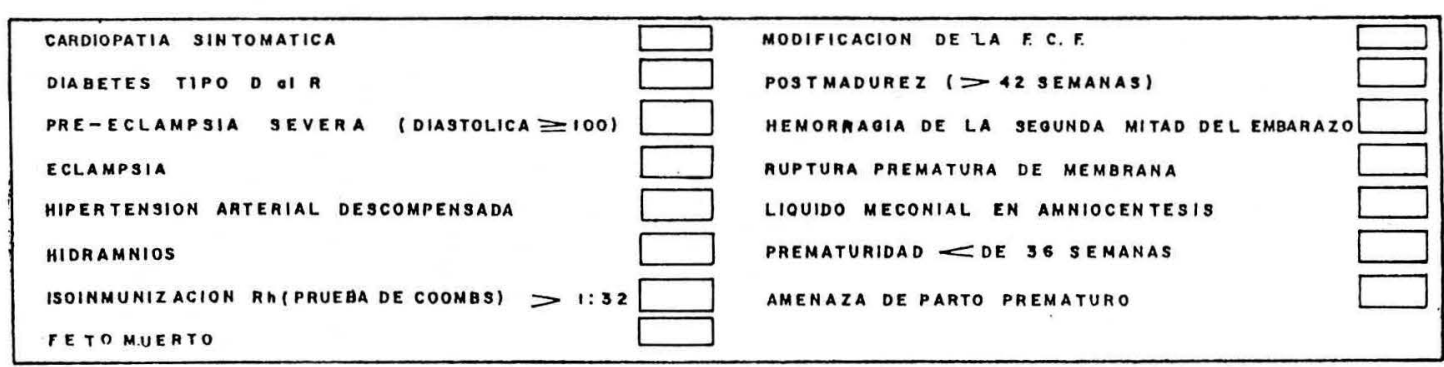

OBSERVACIONES:

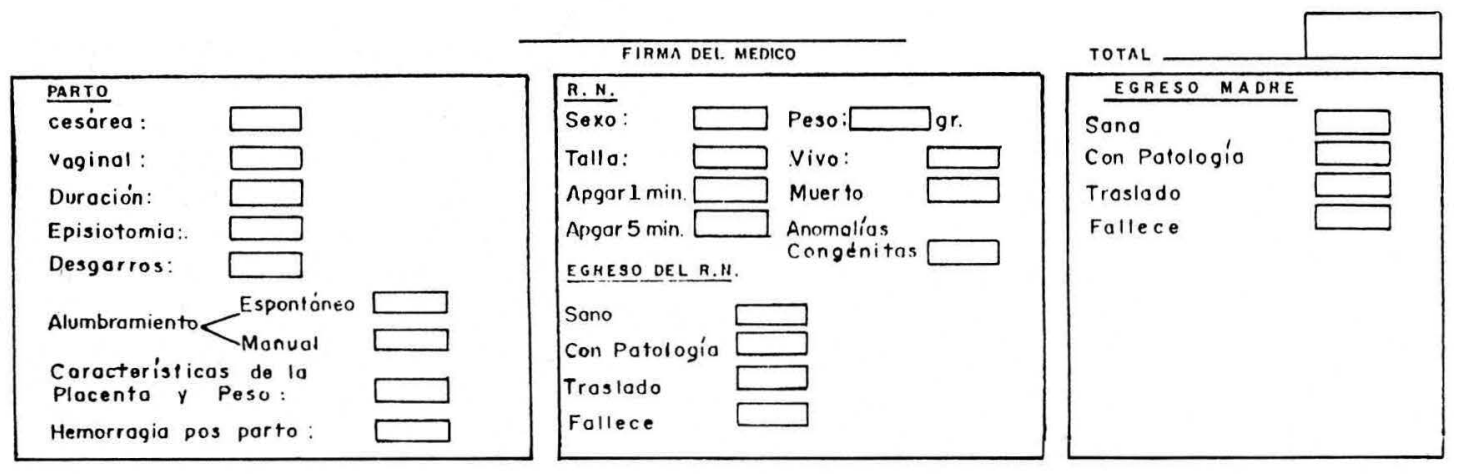


Tabla 6

RELACION ENTRE EL GRADO DE RIESGO MATERNO FETAL Y MORTALIDAD FETAL

\begin{tabular}{|llrrrrr|}
\hline \multirow{2}{*}{ Riesgo } & \multicolumn{1}{c}{ Vivos } & \multicolumn{2}{c|}{ Muertos } & \multicolumn{1}{c|}{ Total } \\
& \multicolumn{1}{c}{ No. Proporción } & No. Proporción No. Proporción \\
\hline Alto & 72 & $80 \%$ & 18 & $20 \%$ & 90 & $100 \%$ \\
Mediano & 8 & $100 \%$ & 0 & $0 \%$ & 8 & $100 \%$ \\
Bajo & 2 & $100 \%$ & 0 & $0 \%$ & 2 & $100 \%$ \\
\hline TOTAL & 82 & & 18 & & 100 & \\
\hline
\end{tabular}

\section{REPRESENTACION DE LA TABLA 6}

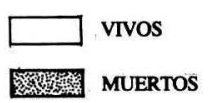

$$
\left[\begin{array}{r}
100 \\
90 \\
-80 \\
70 \\
60 \\
50 \\
40 \\
30 \\
20 \\
10 \\
0
\end{array}\right.
$$
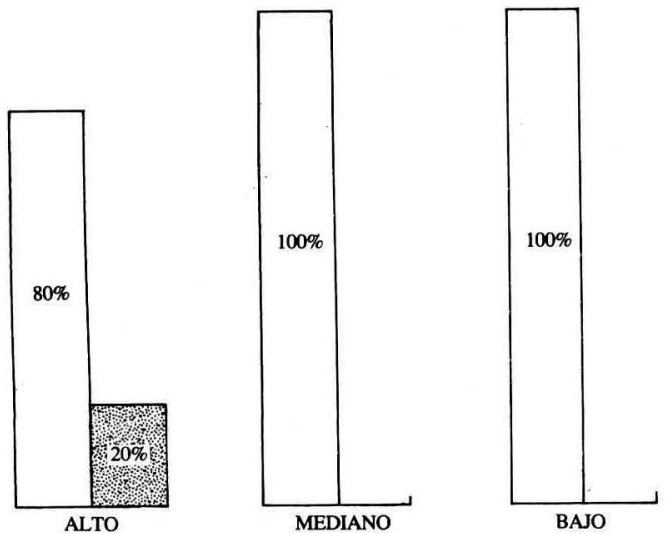

de 0 en el $20 \%$ de los casos; con apgar de 1 a 4 en el $3.3 \%$; de 5 a 7 en el $20 \%$ y de 8 a 10 en el $46.1 \%$. En las de mediano riesgo se obtuvieron recién nacidos con apgar de 0 en el $0 \%$ de los casos; de 1 a 4 en el $0 \%$; de 5 a 7 en el $22.2 \%$; de 8 a 10 en el $77.8 \%$. En las de bajo riesgo se obtuvieron solamente recién nacidos con apgar de 8 a 10 o sea en el $100 \%$ de los casos (Tabla 7).

Riesgo materno y peso del recién nacido. En relación con el riesgo materno y el peso del recién nacido se observó lo siguiente: en las pacientes de alto riesgo se obtuvieron 6 recién nacidos con peso menor de 1.500 grs; 16 con peso entre 1.500 y 2.499 grs; 47 con peso entre 2.500 y 3.499 grs; 8 recién nacidos con peso entre 3.500 y 3.499 grs, y un recién nacido con peso entre 4.000 o más grs.

Entre las de mediano riesgo se encontraron: recién nacidos con peso menor de 1.500 grs, 0 casos; con peso entre 1.500 y 2.499 grs, 5 casos; entre 2.500 y 3.499 grs, 4 casos; con peso entre 3.500 y 3.999 grs, un caso, y entre 4.000 o más grs, ningún caso.

En las pacientes de bajo riesgo sólo se obtuvo un recién nacido con peso entre 2.500 y 3.499 grs (Tabla 8).

Riesgo materno con estado de recién nacido. Relacionando el puntaje de riesgo materno con el estado del recién nacido se observó lo siguiente:

En las pacientes de bajo riesgo (puntaje de I a 4) se encontró un recién nacido sano, ninguno con patología o muerto. Entre las pacientes de mediano riesgo (puntaje de 5 a 9) se encontraron 8 recién nacidos sanos, ninguno con patología o muerto. En las pacientes de alto riesgo (puntaje de 10 o más) se encontraron 53 recién nacidos sanos, 19 con patologías y 18 muertos (Tabla 9).

Tabla 7

RELACION ENTRE GRADO DE RIESGO MATERNO FETAL Y EL APGAR DEL RECIEN NACIDO

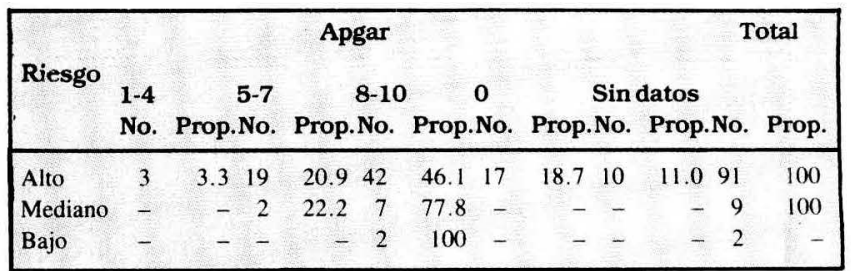

REPRESENTACION DE LA TABLA 7

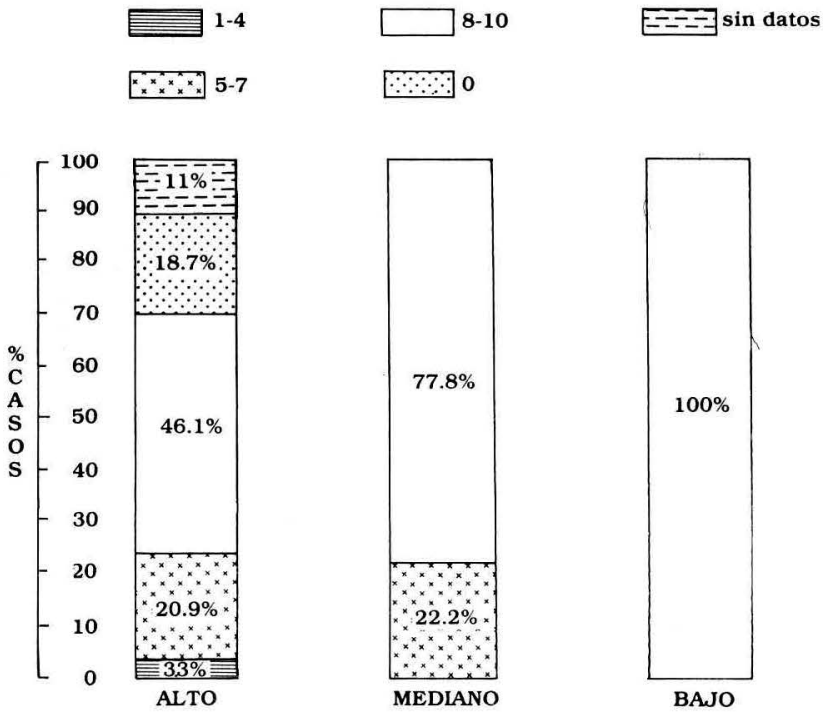

Tabla 8

RELACION ENTRE GRADO DE RIESGO MATERNO FETAL Y PESO DEL RECIEN NACIDO

\begin{tabular}{|c|c|c|c|c|c|c|c|}
\hline \multirow[t]{2}{*}{$\begin{array}{l}\text { Pesoen } \\
\text { gramos }\end{array}$} & \multicolumn{2}{|c|}{ Alto } & \multicolumn{3}{|c|}{$\begin{array}{l}\text { Riesgo } \\
\text { Mediano }\end{array}$} & \multicolumn{2}{|c|}{$\begin{array}{l}\text { Total } \\
\text { 3ajo }\end{array}$} \\
\hline & No. & Propor. & & Propor. 1 & & ropor. No & por. \\
\hline$<150$ & 6 & 100 & - & - & - & - & 100 \\
\hline $1500-2499$ & 16 & 76.2 & 5 & 23.8 & - & - & 100 \\
\hline $2500-3499$ & 47 & 90.4 & 4 & 7.7 & 1 & 1.95 & 100 \\
\hline $3500-3999$ & 8 & 88.9 & 1 & 11.1 & - & - & 100 \\
\hline$\triangle 000$ y más & 1 & 100 & - & - & - & - & 100 \\
\hline
\end{tabular}

Tabla 9

RELACION ENTRE EL PUNTAJE DE RIESGO Y RECIEN NACIDOS

\begin{tabular}{|lllllll|}
\hline \multicolumn{1}{c}{ SANOS - MUERTOS - CON PATOLOGIA } \\
$\begin{array}{l}\text { Puntaje de } \\
\text { riesgo }\end{array}$ & R.N. Sanos & \multicolumn{2}{ll}{ Muertos } & Patología \\
\hline $1-4$ & 1 & $100 \%$ & 0 & $0 \%$ & 0 & $0 \%$ \\
$5-9$ & 8 & $100 \%$ & 0 & $0 \%$ & 0 & $0 \%$ \\
$\geqslant 10$ & 53 & $58.9 \%$ & 18 & $20 \%$ & 19 & $21.1 \%$ \\
\hline
\end{tabular}




\section{REPRESENTACION DE LA TABLA 8}
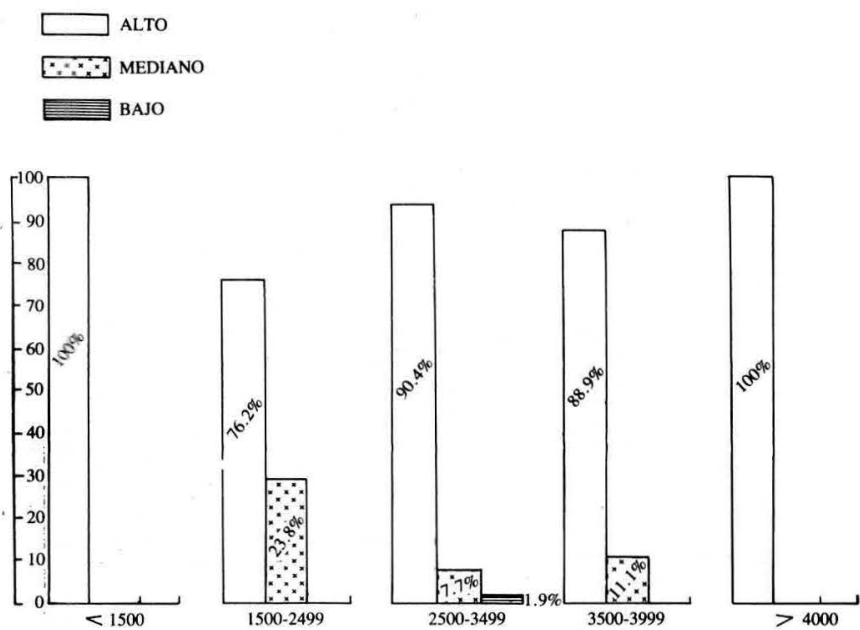

\section{Discusión}

Se pudo demostrar que al aplicar el formulario experimental elaborado en un trabajo científico anterior (5) se puede evaluar en forma cuantitativa el riesgo materno-fetal en el embarazo.

Este formulario permitió clasificar un embarazo como de bajo, mediano o alto riesgo y brindar una atención médica más adecuada y dirigida.

Se demostró mediante el análisis de las tablas de datos y sus respectivas representaciones gráficas, que la aplicación temprana y adecuada de este formulario es eficaz para permitir la clasificación de aquellos embarazos que, por presentar patologías coexistentes, son considerados de riesgo, hecho este por el cual se beneficiaría grandemente el binomio, madre-hijo.
Si este formulario formara parte esencial de todas las historias clínicas gineco-obstétricas elaboradas en la Clínica de Maternidad Rafael Calvo C., puede afirmarse que sería factor contribuyente primordial para la disminución de la morbi-mortalidad materno-fetal.

REPRESENTACION DE LA TABLA 9

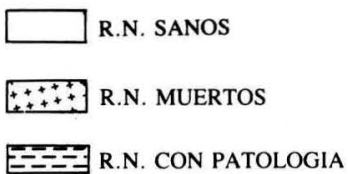

$\left[\begin{array}{r}100 \\ 90 \\ -80 \\ -70 \\ -60 \\ -50 \\ 40 \\ -30 \\ 20 \\ -10 \\ 0\end{array}\right.$
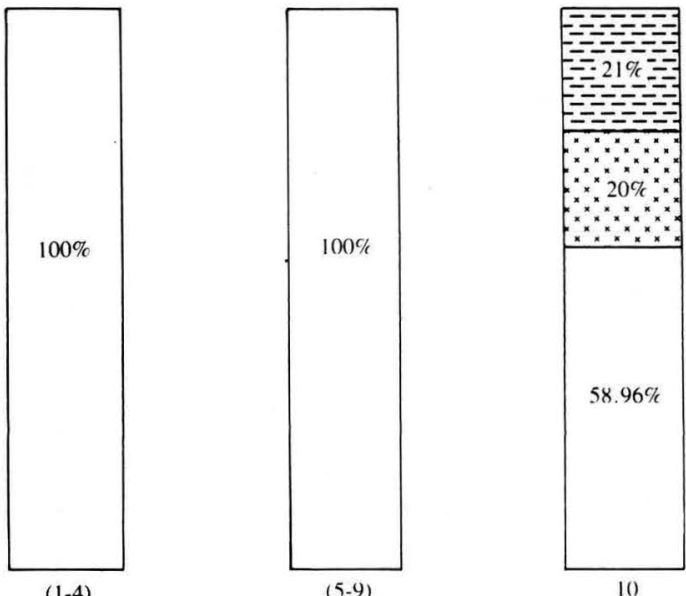

10

\section{BIBLIOGRAFIA}

1. Berkowitz GS. An epidemiologic Study of Preterm Delivery. Am J Epidemiol 1981; 113: 81-92.

2. Centro Latinoamericano de Perinatología. Historia Clínica Perinatal Simplificada.

3. Dougherty CRS, Jones AD. The Determinants of Birth Wcight. Am J Obstet Gynecol 1982; 144-190.

4. Facultad de Medicina de Manizales. Historia Unica Perinatal.

5. Facultad de Medicina. Universidad de Cartagena. Evaluación del Riesgo Materno-Infantil. 1984.

6. Hasbum J. Diagnóstico de Situación perinatal. Rev Col Obstet Ginecol 1983; 34(1): 51-68.

7. Mac Carthy BJ, Schulz KP, Terry JS. Identify ing Neonatal Risk Factors and Predicting Neonatal Deaths in Georgia. Am J Obstet Gynecol 1982; 142-557.
8. Ministerio de Salud. Módulo de Atención Perinatal. 1983.

9. Pasmiño S. Atención Materno-Infantil. Rev Col Obstet Ginecol 1980; 31: 80.

10. Polto JJ, Pérez VL, Pons E, Amado de Realini, T Duhar de Laurino. Embarazo en la adolescencia. Montevideo. Arch Ginecol Obstet 1979; 29(4): 215-222.

11. Schwarcz R. Obstetricia, 3a. ed., Capítulo 1o., 1978, pág. 14, 315.

12. Soto Yances A, Merlano Elsy y col. Evaluación del Riesgo MaternoFetal. Dpto. de Obst. y Ginecol. Universidad de Cartagena. Presentado al XVI Congreso Colombiano de Obstetricia y Ginecología. Cali, 1985. 